Gut, 1981, 22, 1042-1045

Case report

\title{
Detection of pulmonary arteriovenous fistulae in patient with cirrhosis by contrast 2D echocardiography
}

\author{
C R K HIND* AND C M WONG \\ From the Cardiac Department, Brompton Hospital, London.
}

SUMMARY A case of multiple pulmonary arteriovenous fistulae associated with cirrhosis of the liver is described. The diagnosis was made by contrast two-dimensional echocardiography and confirmed by cardiac catheterisation and pulmonary angiography. As it is a sensitive and noninvasive technique, contrast two-dimensional echocardiography may be a useful screening test in cyanotic patients with liver disease in whom the presence of pulmonary fistulae is suspected.

The association of hepatic cirrhosis with cyanosis, finger clubbing, and arterial oxygen desaturation has been recognised for many years. ${ }^{1}$ It is thought to be due to multiple pulmonary arteriovenous fistulae which have previously been demonstrated only by examination of micropaque arteriograms of postmortem lungs. ${ }^{2}$ We report a case of cirrhosis in whom contrast two-dimensional echocardiography enabled a definite diagnosis of pulmonary arteriovenous fistulae to be made in life.

\section{Case report}

The patient, a 15 year old Greek boy, was admitted to Brompton Hospital for investigation of central cyanosis. Childhood and development had been normal with no past medical history of note. Two years previously his parents had noted purple lips, worse on exertion, and for three months before admission he had been an inpatient in Greece for investigation of fever and dark urine. Blood cultures had grown Staphylococcus epidermidis, for which he received a number of different antibiotics intravenously. He was referred to England for additional investigation. His symptoms on admission here were of some dyspnoea and tiredness on extreme exertion.

On examination, he was tall $(1.89 \mathrm{~m})$, thin boy

*Address for correspondence: Dr Charles Hind, Department of Medicine, Hammersmith Hospital, Du Cane Road, London, W12 0HS

Received for publication 1 June 1981 with normal secondary sexual characteristics. He was centrally cyanosed with clubbing of the fingers and toes. There were a few spider naevi on his upper limbs. Examination of the respiratory system was normal. On cardiovascular examination, his pulse was $90 / \mathrm{min}$ regular, blood pressure $130 / 80 \mathrm{~mm} \mathrm{Hg}$. Apical impulse and venous pressure were normal. The first heart sound was normal; the pulmonary component of the second heart sound was loud and a pulmonary ejection click was present, but there was no clinical evidence of pulmonary hypertension or right heart failure. Abdominal examination revealed slight hepatomegaly $(2 \mathrm{~cm})$ and moderate splenomegaly ( $4 \mathrm{~cm}$ below the costal margin).

\section{INVESTIGATIONS}

Haemoglobin and WBC within normal limits. ESR was $62 \mathrm{~mm} / \mathrm{h}$. Prothrombin ratio was $1 \cdot 5$. Total bilirubin was $36 \mu \mathrm{mol} / \mathrm{l}$, and conjugated $21 \mu \mathrm{mol} / \mathrm{l}$. Alkaline phosphatase was 374 IU/l. Gamma glutyamytransferase was $28 \mathrm{IU} / \mathrm{l}$. Aspartate transaminase was $28 \mathrm{IU} / \mathrm{l}$. Other investigations including plasma urea and electrolytes, creatinine, blood glucose, total protein, albumin, calcium, and phosphate were all within normal limits, as were $\mathrm{HBsAg}$, alpha-1 antitrypsin level, serum copper, and an antibody screen. Repeated blood cultures were negative.

Radiograph of the chest showed a normal-sized heart and normal proximal pulmonary arteries, but there was a suggestion of increased pulmonary vasculature in the periphery of the lung. The ECG was normal. 
Pulmonary function tests showed normal lung volumes and expiratory flows. Carbon monoxide transfer factor (DLCO) was reduced to $40 \%$ of the predicted value. At rest on air, $\mathrm{PaO}_{2}$ was $6.3 \mathrm{kPa}$ $(47.1 \mathrm{~mm} \mathrm{Hg})$, and $\mathrm{PaCO}_{2}$ was $3.95 \mathrm{kPa}(29.5 \mathrm{~mm}$ $\mathrm{Hg}$ ). On $100 \%$ oxygen, $\mathrm{PaO}_{2}$ was $46.1 \mathrm{kPa}(346 \mathrm{~mm}$ $\mathrm{Hg}$ ). On exercise, $\mathrm{PaO}_{2}$ was $5.1 \mathrm{kPa}(37.9 \mathrm{~mm} \mathrm{Hg})$, and $\mathrm{PaCO}_{2}$ was $3.0 \mathrm{kPa}(22.5 \mathrm{~mm} \mathrm{Hg})$. Maximum work load achieved was $100 \mathrm{kpm}$.

The $\mathbf{M}$ mode echocardiogram was normal. Twodimensional echocardiography showed normal intracardiac anatomy and no evidence of atrial or ventricular septal defect. Contrast two-dimensional echocardiogram was performed. An injection of 10 $\mathrm{ml}$ normal saline was given rapidly into the right antecubital vein by hand. The progress of the contrast was recorded with the transducer in the parasternal four-chamber position (Fig. 1). After the injection, the right atrium and ventricle were opacified (Fig. 2), but the left heart chambers were free of contrast until three cardiac cycles $(3 \mathrm{~s})$ later when contrast was seen to enter the left atrium and left ventricle (Fig. 3). These findings are compatible with a right-to-left shunt through the lung vasculature rather than at atrial or ventricular level.

Cardiac catheterisation showed normal right atrial, ventricular, and pulmonary artery pressures. Total pulmonary blood flow using Direct Fick method was $13 \mathrm{l} / \mathrm{min}$. A pulmonary arteriogram showed very early venous filling with blotchy

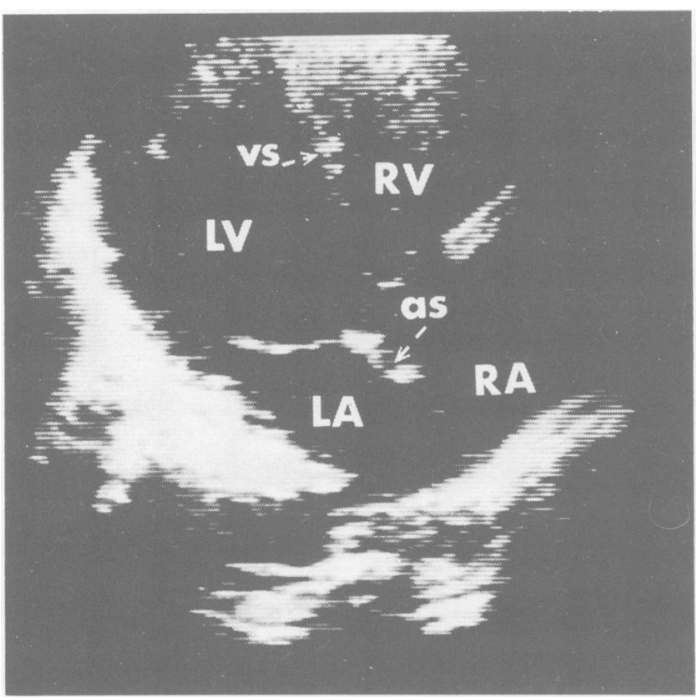

Fig. 1 Parasternal four chamber view of heart. LA: left atrium. $L V$ : left ventricle: $R A$ : right atrium. $R V$ : right ventricle. $A S$ : atrial septum. VS: ventricular septum. shadows at the bases suggesting precapillary shunting.

Liver biopsy showed cirrhosis with no inflammatory activity.

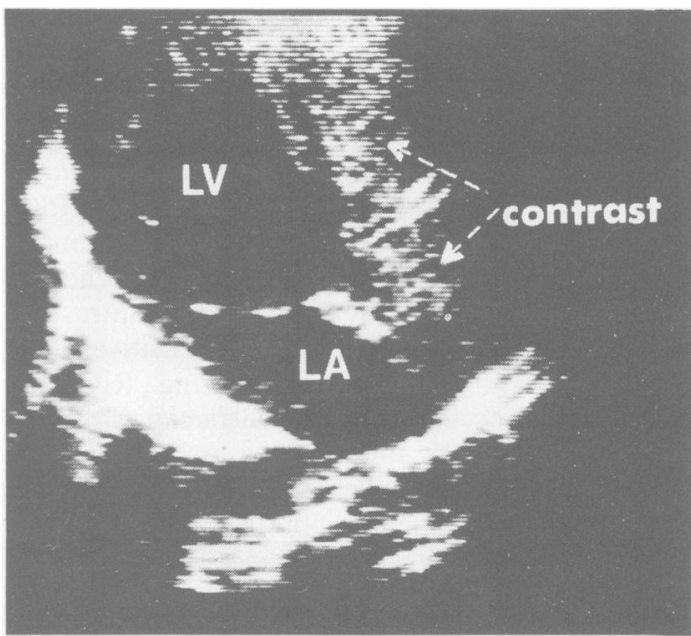

Fig. 2 Appearance of contrast in right atrium and right ventricle. Left heart chambers are free of contrast at this stage, excluding right-to-left shunt at atrial level. LA: left atrium. $L V$ : left ventricle.

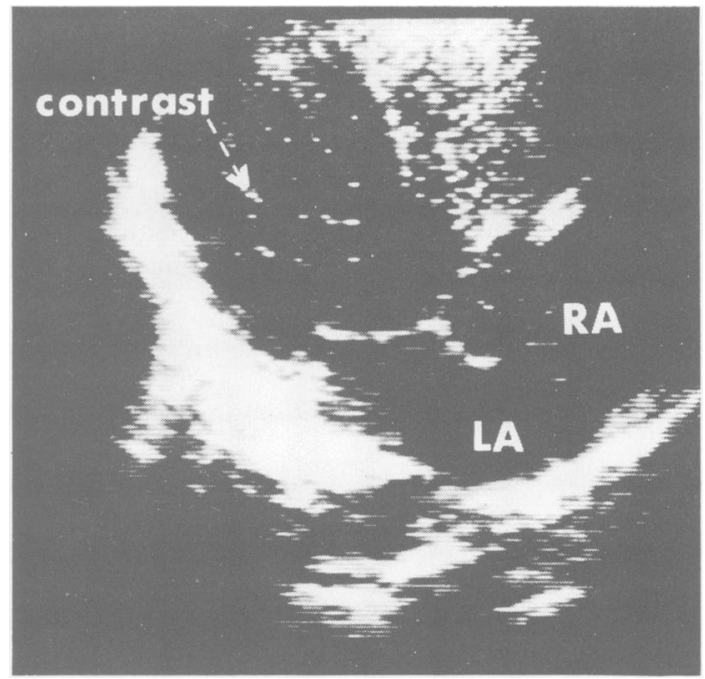

Fig. 3 Parasternal four chamber view three seconds after opacification of right ventricle with contrast. Contrast can now be seen in left ventricle after its appearance in left atrium. RA : right atrium. LA : left atrium. 


\section{Discussion}

The presence of multiple microscopic pulmonary arteriovenous fistulae cannot be identified on radiographs of the chest or by lung biopsy specimens. ${ }^{1}$ Definitive diagnosis can be made by pulmonary arteriography, which has shown a dense spongy appearance during the capillary phase in a few cases, but has been normal in others. ${ }^{3}$ Similarly, abnormal lung perfusion scans have been noted in some but not all cases. ${ }^{4}$

The diagnosis of multiple microscopic pulmonary $\mathrm{AV}$ fistulae may be made non-invasively by contrast echocardiography. Detection of a large pulmonary arteriovenous fistulae using simple $\mathbf{M}$ mode echocardiography was described by Shub et al. ${ }^{5}$ Contrast $\mathbf{M}$ mode and 2D echo are useful and sensitive techniques in detecting right-to-left shunting. Right-toleft shunts can occur at atrial, ventricular, or great artery level. With the shunt at the atrial or ventricular level, contrast can be seen passing across the atrial or ventricular septum in the parasternal or apical four-chamber views. Opacification of the left heart chambers by contrast occurs immediately after opacification of those of the right heart. When the shunt is at the great artery level, contrast does not appear in either the left atrium or ventricle. A number of contrast agents may be used, including normal saline, $5 \%$ dextrose, or indocyanine green. The contrast effect is thought to arise from reflection of ultrasound by microcavitations or microbubbles present in the injected solution. Normally, contrast is cleared after passing through normal pulmonary alveolar capillaries, ${ }^{5}$ possibly by dispersion of microcavitations during their passage through small vessels or contact with alveoli. The appearance of contrast in the left heart chambers after the passage through the lung is thus abnormal and indicates the presence of AV connections bypassing the alveoli. Shunting of this sort due to abnormal AV connections is distin- guished from shunting at either the atrial or ventricular levels by a delay in the appearance of contrast in the left heart chambers. This delay, usually 2-3 cardiac cycles, represents the time taken for the contrast to pass through the abnormal AV connections. Although the delayed appearance of contrast in the left atrium indicates an abnormal vascular pathway in the lung, it does not differentiate a single large pulmonary AV fistula from multiple microscopic AV connections. The former diagnosis can readily be made from a plain radiograph of the chest or pulmonary angiogram. Contrast echocardiography may thus be a useful non-invasive technique for detecting the presence of pulmonary AV fistulae too small to be demonstrated by other methods.

We would like to thank Dr D G Gibson for reading the manuscript and for his helpful suggestions.

\section{References}

${ }^{1}$ Rydell R, Hoffbauer FW. Multiple pulmonary arteriovenous fistulae in juvenile cirrhosis. Am J Med 1956; $21: 450-60$.

${ }^{2}$ Berthelot P, Walker JG, Sherlock S, Reid L. Arterial changes in the lungs in cirrhosis of the lung - lung spider naevi. $N$ Engl J Med 1966; 274:291-98.

${ }^{3}$ Hansoti RC, Shah NJ. Cirrhosis of liver simulating congenital cyanotic heart disease. Circulation 1966; 33:71-77.

${ }^{4}$ Stanley N, Ackrill P, Wood J. Lung perfusion scanning in hepatic cirrhosis. Br Med J 1972; 4:639-43.

${ }^{5}$ Shub C, Tajik AJ, Seward JB, Dines DE. Detecting intrapulmonary right-to-left shunt with contrast echocardiography. Mayo Clin Proc 1976; 51:81-84.

${ }^{6}$ Gramiak R, Shah PM, Kramer DH. Ultrasound cardiography contrast studies in anatomy and function. Radiology 1969; 92:939-48.

${ }^{7}$ Weiss EW, Gasul BM. Pulmonary arteriovenous fistula and telangiectasia. Ann Intern Med 1954; 41:989-1002. 